\title{
Results of a moderate sedation program with propofol for transesophageal echocardiography performed by non-anesthesiologist professionals.
}

\author{
Chi-Hion Li ${ }^{1}$, Violeta González-Salvado ${ }^{2}$, Ester Bertolí1 ${ }^{1}$, David Viladés ${ }^{1}$, Martín Descalzo ${ }^{1}$, \\ Francesc Carreras ${ }^{3}$, Rubén Leta ${ }^{1}$, Juan Cinca ${ }^{3}$, Helena Capellades ${ }^{4}$, Luisa Cueva ${ }^{1}$, and \\ Pilar Paniagua ${ }^{1}$ \\ ${ }^{1}$ Hospital de la Santa Creu i Sant Pau \\ ${ }^{2}$ Hospital Clínico Universitario de Santiago de Compostela Servicio de Cardiología \\ ${ }^{3}$ Universitat Autònoma de Barcelona \\ ${ }^{4}$ Hospital de Sant Joan Despí Moisès Broggi
}

October 20, 2020

\begin{abstract}
Introduction and objectives The advantages of sedation in patients undergoing transesophageal echocardiography (TEE) are well established, but the increasing number of TEE studies may limit the capacity of Anesthesiology services. In this study we analyze the effectiveness and safety of a moderate sedation program carried out by non-anesthesiologist professionals (NAP). Methods Descriptive and prospective study that included all consecutive patients undergoing a TEE procedure under moderate sedation by NAP and by anesthesiologists (AP) from September 2018 to September 2019. Patients were selected according to a specific algorithm agreed upon with the Anesthesiology department. We analyze current indications for TEE, complication rates, and recovery times associated with sedation. Results We performed 267 procedures in 252 patients (54\% male, $69 y$ ). Main indications were screening or monitoring of surgical and percutaneous cardiac interventions (47.9\%), endocarditis (28.5\%) and stroke (20.6\%). Patients in the NAP group were younger (59 vs 71 yo), less hypertensive ( $43 \%$ vs $61 \%$ ), with less lung disease ( $4 \%$ vs $24 \%$ ), lower risk of difficult airway (1\% vs $22 \%$ ) and lower comorbidity (ASA scale [?]III $13 \%$ vs $77 \%$ ). Complications were more frequent in the AP group (26\% vs $9 \%$ ), mainly mild respiratory (19\% vs $9 \%$ ). Multivariate adjustment showed events were associated with the presence of lung disease and higher ASA degree. Recovery time was longer in the AP sedation group (20min vs $15 \mathrm{~min}$ ). Conclusions TEE indications are changing and involve larger and more complex studies. Moderate sedation with propofol performed by NAP is safe in selected patients, with adequate recovery times.
\end{abstract}

Title: Results of a moderate sedation program with propofol for transesophageal echocardiography performed by non-anesthesiologist professionals.

\section{Running head: Propofol TEE sedation for non-anesthesiologists}

\section{Authors}

Chi-Hion Li ${ }^{*}, 1,2$, Violeta González-Salvado ${ }^{3}$, Ester Bertolí ${ }^{1}$, David Viladés ${ }^{1}$, Martín Descalzo ${ }^{1}$, Francesc Carreras $^{2}$, Rubén Leta ${ }^{1}$, Juan Cinca ${ }^{2}$, Helena Capellades ${ }^{4}$, Luisa Cueva ${ }^{5}$, Pilar Paniagua ${ }^{5}$.

${ }^{1}$ Cardiology department. Hospital de la Santa Creu i Sant Pau. Barcelona.

${ }^{2}$ Medicine department. Universitat Autònoma de Barcelona. Barcelona. 
3 Cardiology department. Hospital Clínico Universitario de Santiago. CIBERCV, Santiago de Compostela (A Coruña)

${ }^{4}$ Cardiology department. Hospital Moisès Broggi. Sant Joan Despí. Barcelona.

${ }^{5}$ Anesthesiology department. Hospital de la Santa Creu i Sant Pau, Barcelona.

*Corresponding author:CH.PedroLi@gmail.com.

Hospital de la Santa Creu i Sant Pau.

C. Sant Antoni Maria Claret, 167.

08025 Barcelona.

Phone: +34

Fax number:

Hosted file

English_manuscript_final.pdf available at https://authorea.com/users/368827/articles/ 487843-results-of-a-moderate-sedation-program-with-propofol-for-transesophagealechocardiography-performed-by-non-anesthesiologist-professionals

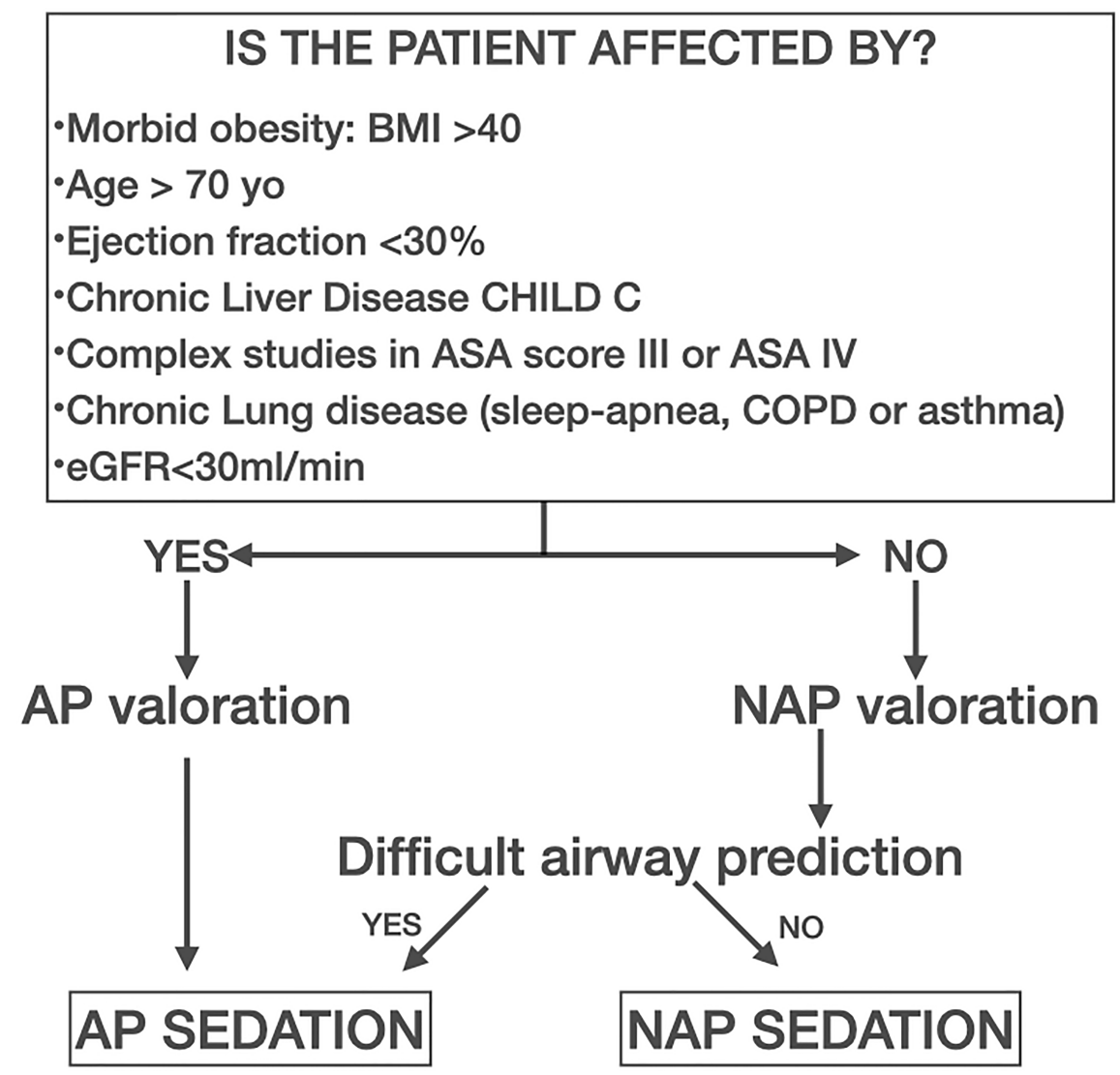

